\title{
Aplicação do Open-Book Accounting (OBA) na perspectiva dos fornecedores de autopeças do Brasil
}

\begin{abstract}
Vinícius Almeida Cordeiro
Graduação em andamento em Ciências Contábeis pela Universidade Federal de Uberlândia - UFU Av. João Naves de Ávila, 2121 - 1F. Santa Mônica. Uberlândia/MG. CEP: 38.408-100 E-mail: viniciusalmeidacordeiro@hotmail.com

Lara Cristina Francisco de Almeida Fehr Doutorado em Controladoria e Contabilidade pela Faculdade de Economia e Administração da Universidade de São Paulo - FEA/USP Professora da Universidade Federal de Uberlândia - UFU Av. João Naves de Ávila, 2121 - 1F. Santa Mônica. Uberlândia/MG. CEP: 38.408-100 E-mail: larafehr@ufu.br

Ana Clara Gama Oliveira Graduação em andamento em Ciências Contábeis pela Universidade Federal de Uberlândia - UFU Av. João Naves de Ávila, 2121 - 1F. Santa Mônica. Uberlândia/MG. CEP: 38.408-100 E-mail: anaclara1370@gmail.com

Anderson Martins Cardoso Mestrado em Controladoria pela Universidade Federal de Uberlândia - UFU Av. João Naves de Ávila, 2121 - 1F. Santa Mônica. Uberlândia/MG. CEP: 38.408-100 E-mail: andersonmar@yahoo.com.br

Marcelo Tavares Doutorado em Agronomia pela Universidade de São Paulo - ESALQ/USP Professor da Universidade Federal de Uberlândia - UFU Av. João Naves de Ávila, 2121 - 1F. Santa Mônica. Uberlândia/MG. CEP: 38.408-100 E-mail:mtavares@ufu.br
\end{abstract}

\section{RESUMO}

As empresas têm estabelecido novas formas de relacionamento em resposta ao ambiente competitivo e globalizado no qual estão inseridas. Essa realidade exige que as empresas apliquem uma gestão de custos que vai além dos limites de uma única empresa. É nesse contexto que se inserem o Open-Book Accounting (OBA) e a Gestão de Custos Interorganizacionais (GCl), que possuem o propósito de gerar e/ou manter vantagem competitiva. O objetivo deste estudo foi identificar como é aplicado o OBA na perspectiva dos fornecedores de autopeças no Brasil. Utilizou-se a estratégia quantitativa, sendo uma pesquisa descritiva. Foram aplicados questionários aos fornecedores de autopeças de montadoras brasileiras. Os resultados evidenciaram que 
Aplicação do Open-Book Accounting (OBA) na perspectiva dos fornecedores de autopeças do

Brasil

Vinícius Almeida Cordeiro, Lara Cristina Francisco de Almeida Fehr, Ana Clara Gama Oliveira, Anderson Martins Cardoso, Marcelo Tavares

- OBA é aplicado de maneira unilateral, dos fornecedores para as montadoras, com baixo nível de detalhe e com baixa frequência. Os fornecedores indicaram, em sua maioria, compartilhar suas informações por ser uma exigência das montadoras. Os achados evidenciaram também que não há incentivo para o compartilhamento de informações, o que não está alinhado com a literatura na área. Conclui-se que o OBA mostra-se um processo fraco no setor automotivo, o que pode estar relacionado à forma de sua aplicação, unilateralmente, gerando falta de confiança pelo risco de oportunismo e afetando, por exemplo, o nível de detalhe e a frequência do compartilhamento das informações. Com isso, essas empresas perdem a oportunidade de aplicar uma adequada gestão de custos interorganizacionais que os ajudaria a gerar e/ou manter vantagem competitiva.

Palavras-chave: Gestão de Custos Interorganizacionais. Open-Book Accounting. Setor Automotivo. Autopeças.

\section{Application of Open-Book Accounting (OBA) from the perspective of Brazilian} auto parts suppliers

\section{ABSTRACT}

Companies have established new forms of relationship in response to the competitive and globalized environment to which they operate. This demands companies to apply cost management that goes beyond the limits of a single company. It is in this context that Open-Book Accounting (OBA) and Interorganizational Cost Management (GCl) are inserted, which have the purpose of generating and/or maintaining competitive advantage. The objective of this study was to identify how OBA is applied from the perspective of auto parts suppliers in Brazil. This is a descriptive research as quantitative strategy was used. Questionnaires were applied to the auto parts suppliers of Brazilian automakers. The results showed that OBA is applied unilaterally, from suppliers to automakers, with low level of detail and low frequency. Most suppliers indicated that they share their information because it is a requirement of the automakers. The findings also showed that there is no incentive to share information, which is not in line with the literature in the area. It can be concluded that OBA proves to be a weak process in the automotive sector, which may be related to its application unilaterally, generating a lack of confidence due to the risk of opportunism and affecting, for example, the level of detail and frequency of information sharing. As result, these companies miss the opportunity to apply proper inter-organizational cost management that would help them generate and/or maintain competitive advantage.

Keywords: Interorganizational Cost Management. Open-Book Accounting. Automotive industry. Auto parts. 
Aplicação do Open-Book Accounting (OBA) na perspectiva dos fornecedores de autopeças do

Brasil

Vinícius Almeida Cordeiro, Lara Cristina Francisco de Almeida Fehr, Ana Clara Gama Oliveira, Anderson

Martins Cardoso, Marcelo Tavares

\section{INTRODUÇÃO}

Com o avanço da globalização e o consequente aumento das pressões competitivas, empresas inseridas em relacionamentos estreitos têm implementado práticas colaborativas para conseguirem manter-se nesse ambiente. Para tanto, se estruturam em cadeias, de onde passam a depender cada vez mais da eficiência de seus parceiros de negócios (Martins, Souza Filho, \& Di Serio, 2011).

O ambiente competitivo exige das empresas a busca contínua pela redução de custos, e abordagens com foco na gestão dos custos, como é o caso da Gestão de Custos Interorganizacionais (GCl) e do Open-Book Accounting (OBA), ganham relevância. DhaifAllah, Md Auzair, Maelah e Ismail (2016) afirmam que o OBA e a GCI melhoram os relacionamentos baseados em parcerias entre empresas, principalmente quando subsistem salvaguardas relacionais, que servem para mitigar eventuais comportamentos oportunistas (Di \& Wang, 2017).

Tais ferramentas de gestão de custos são úteis para controlar as atividades realizadas por fornecedores externos, reduzir custos, melhorar a eficiência, gerar benefícios mútuos, criar e/ou manter vantagem competitiva e outros (Mouritsen, Hansen, \& Hansen, 2001; Kajüter \& Kulmala, 2005; Bhimani, Souza, \& Rocha, 2011; Romano \& Formentini, 2012; Faria, Soares \& Rocha, 2013; Ellström \& Larsson, 2017).

Fehr (2015) define o OBA como sendo um "processo de compartilhamento de informações, a princípio sigilosas, relativas a custos, processos e/ou atividades, entre partes em um relacionamento, com o objetivo de otimizar a gestão de custos". Para Kajüter e Kulmala (2005), o foco do OBA é aumentar a eficiência da $\mathrm{GCl}$, potencializando a redução adicional de custos por meio de ações conjuntas entre compradores e fornecedores, e Suomala, Lahikainen, Lyly-Yrjänäinen \& Paranko (2010) confirmam que o OBA é a tecnologia mais reconhecida na aplicação da GCl.

Nesse contexto, se insere a indústria automobilística que vem historicamente apresentando relevantes economias de custos (Carr \& $\mathrm{Ng}$, 1995), sendo um dos 
Aplicação do Open-Book Accounting (OBA) na perspectiva dos fornecedores de autopeças do

Brasil

Vinícius Almeida Cordeiro, Lara Cristina Francisco de Almeida Fehr, Ana Clara Gama Oliveira, Anderson

Martins Cardoso, Marcelo Tavares

principais setores de aplicação do OBA em relacionamentos entre fornecedores e compradores, tendo origem na década de 1990 com a disseminação do modelo de produção enxuta japonês (Kajüter \& Kulmala, 2005). Ademais, o setor automotivo é precursor de várias técnicas gerenciais e inovações tecnológicas, as quais são utilizadas por empresas de diversos setores, o que demonstra sua influência às empresas de um modo geral (Abinajm-Filho, 2011).

Dados da Associação Nacional dos Fabricantes de Veículos Automotores [ANFAVEA] $(2017$; 2018) mostram que a indústria automotiva, em 2015, alcançou um faturamento de 59,1 bilhões de dólares, gerou tributos diretos no montante de 39,7 bilhões de reais e participou com $22 \%$ do PIB industrial nacional e, em 2016, gerou cerca de 1,3 milhões de empregos diretos e indiretos no Brasil. Já em 2017, a produção de veículos no Brasil fechou com alta de 25,2\%; no total, foram feitos 2.699 .672 carros, caminhões e ônibus.

Diante do exposto e considerando-se a necessidade da gestão de custos além dos limites de uma única empresa e a importância do setor automotivo em termos de pioneirismo na aplicação de técnicas interorganizacionais e de sua representatividade econômica e social, formulou-se a seguinte questão-problema: Como é aplicado o OBA na percepção dos fornecedores de autopeças do Brasil? Assim, o objetivo geral foi identificar como é aplicado o OBA na perspectiva dos fornecedores de autopeças no Brasil.

Com o intuito de atingir o objetivo geral, foram delineados os seguintes objetivos específicos: (i) verificar em que contexto o OBA é aplicado pelas empresas investigadas; e (ii) identificar e analisar as dimensões do OBA presentes nas empresas analisadas. Pelo exposto nas análises da seção 4, o objetivo geral e os objetivos específicos foram alcançados.

Entender como ocorre o processo de compartilhamento de informações entre empresas na cadeia de suprimentos do setor automotivo revela-se importante pelo tamanho desse setor. Comparando-se com a gestão de custos tradicional em empresas 
Aplicação do Open-Book Accounting (OBA) na perspectiva dos fornecedores de autopeças do

Brasil

Vinícius Almeida Cordeiro, Lara Cristina Francisco de Almeida Fehr, Ana Clara Gama Oliveira, Anderson

Martins Cardoso, Marcelo Tavares

individuais, Fehr (2015) observa que o OBA proporciona novas oportunidades para a gestão, assim como para a redução de custos por meio de esforços entre os membros de uma mesma cadeia.

Windolph e Moeller (2012) mostraram que a maior parte das pesquisas na área abordam a extensão da implementação e do uso do OBA, mas no Brasil existem poucos trabalhos sobre o assunto, tais como: Souza (2008), Aguiar, Rezende e Rocha (2008), Camacho (2010), Faria et al. (2013), Santos et al. (2015), Fehr (2015), Fernandes, Fernandes e Fernandes (2015), Kanimura, Mattos e Scur (2016), sendo que parte deles trata mais da $\mathrm{GCl}$ que do OBA.

Por fim, Suomala et al. (2010) e Faria et al. (2013) também destacam a necessidade de pesquisas empíricas nessa área. Com isso, este estudo justifica-se e contribui com a literatura, mostrando como o OBA é praticado no setor automotivo e evidenciando as dificuldades na sua aplicação, as quais refletem no seu funcionamento e desempenho. Espera-se também contribuir com os gestores das empresas estudadas, com a indicação de melhorias que podem aperfeiçoar a aplicação do OBA e gerar vantagem competitiva para as mesmas.

Este trabalho está estruturado em quatro seções além desta. A segunda seção apresenta o referencial teórico, em que são abordados aspectos sobre relacionamentos interorganizacionais no setor automotivo, características e dimensões do OBA, seu relacionamento com a $\mathrm{GCl}$ e os estudos correlatos. Na terceira seção, têm-se os aspectos metodológicos utilizados no desenvolvimento desta pesquisa. Já na quarta seção, apresentam-se a análise e as discussões dos resultados. Por fim, na quinta seção, são abordadas as considerações finais. 
Aplicação do Open-Book Accounting (OBA) na perspectiva dos fornecedores de autopeças do

Brasil

Vinícius Almeida Cordeiro, Lara Cristina Francisco de Almeida Fehr, Ana Clara Gama Oliveira, Anderson

Martins Cardoso, Marcelo Tavares

\section{REFERENCIAL TEÓRICO}

\subsection{Relacionamento Interorganizacional no Setor Automotivo}

Em um contexto onde a eficiência e a competitividade estão fortemente presentes, uma logística eficiente entre fornecedor e montadora tem tido importante destaque nesse setor. Dado um conjunto de circunstâncias, o setor automobilístico tem adotado novas parcerias estratégicas, visando a um relacionamento mais estável e maior colaboração de longo prazo entre as partes da cadeia de suprimentos (Marini, 2003; Vanalle \& Salles, 2011).

Neste cenário, Faria et al. (2013) consideram que a indústria automobilística foi forçada a adotar novas estratégias para conseguir realizar seu trabalho de forma conjunta com diferentes organizações, criando entre elas vínculos focados na cooperação e na eliminação de fronteiras, gerando maior rapidez de entrega, flexibilidade de volume, diferenciação do produto e uma consequente redução dos custos de produção e otimização de processos.

Martins, Souza Filho e Pereira (2012) mostram que, devido à internacionalização, um intenso processo de transformações nas plantas de fábrica se iniciou no Brasil, e com isso houve maior aproximação entre fornecedores e montadoras. Tal dinâmica não se limitou ao nível de relacionamentos, mas também à aproximação geográfica, pois esta proporcionava uma otimização dos recursos, levando em conta a logística de entrega just-in-time.

Assim, surgem nas montadoras os condomínios industriais, onde determinados membros da cadeia produtiva constroem suas instalações dentro da própria montadora, fornecendo componentes diretamente à linha de montagem. Outro modelo é o de consórcio modular, onde a montadora é quem fica responsável pela planta e também pela linha de montagem final, além de coordenar e testar os veículos. Dessa forma, seus fornecedores modulares são responsáveis por realizar a montagem prévia 
Aplicação do Open-Book Accounting (OBA) na perspectiva dos fornecedores de autopeças do

Brasil

Vinícius Almeida Cordeiro, Lara Cristina Francisco de Almeida Fehr, Ana Clara Gama Oliveira, Anderson

Martins Cardoso, Marcelo Tavares

daquele respectivo módulo diretamente na linha de montagem final da montadora (Vanalle \& Salles, 2011).

Em pesquisa recente, Costa e Henkin (2016) caracterizam como estratégia competitiva comum de otimização de desempenho das indústrias do setor automotivo brasileiro a atualização das suas plantas e investimentos em economias de escala e escopo que, facilitadas pela organização modular de produção, permitem a montagem de diferentes modelos de veículos na mesma montadora.

Nos anos de 1990 a 2000, Santos (2001) também percebera diversas melhorias de processos de forma coletiva em diferentes montadoras, entre elas: instalação de economias de escalas, flexibilização nos processos da produção, melhorias tecnológicas e plantas modernas, treinamentos internos para eficiência de operação, reorganização da rede de fornecimento.

Neste contexto interorganizacional cooperativo e competitivo, Grafton e Mundy (2017) verificaram que, na indústria automobilística, contratos baseados no relacionamento mais estreito muitas vezes substituem contratos formais. Firmados principalmente na confiança, esses contratos, por vezes, apresentam maiores riscos para a montadora e sacrificam interesses de curto prazo, porém possuem a intenção de assegurar interesses de longo prazo.

\subsection{Open Book Accounting}

O OBA é uma ferramenta da $\mathrm{GCl}$. De modo geral, a $\mathrm{GCl}$ é aplicada com a intenção de atingir metas de custeio, favorecer o gerenciamento e, até mesmo, a investigação de custos interorganizacionais, sendo o OBA onde se processa a divulgação desses dados de custos e o compartilhamento de informações contábeis de gestão em geral (DhaifAllah et al., 2016).

Para Kulmala, Paranko e Uusi-Rauva (2002), a principal razão para o compartilhamento de informações, ao nível do OBA, é obter vantagens competitivas e não somente a redução dos custos, sendo uma relação de "ganha-ganha" em que 
Aplicação do Open-Book Accounting (OBA) na perspectiva dos fornecedores de autopeças do

Brasil

Vinícius Almeida Cordeiro, Lara Cristina Francisco de Almeida Fehr, Ana Clara Gama Oliveira, Anderson

Martins Cardoso, Marcelo Tavares

todas as partes envolvidas saem ganhando; e também que as grandes empresas estão concentrando seus negócios principais e terceirizando o restante, o que faz com que o sucesso delas dependa cada vez mais da habilidade que elas possuem em controlar 0 que acontece externamente.

Sadeghi e Jokar (2014) definem o OBA como a abertura dos detalhes de custos entre diversas empresas parceiras, sendo que o nível de detalhamento em que esses dados serão divulgados estão diretamente ligados com a confiança, o comprometimento, características do produto e do processo de troca entre todas as partes do relacionamento.

Fehr (2015) pondera que este processo não ocorre de forma espontânea. O objetivo principal continua sendo a otimização dos custos, porém a base dessa relação não é apenas a confiança entre as partes, mas também a busca por benefícios individuais, como, por exemplo, reivindicar reajustes de preços. Portanto, o OBA pode anular a confiança entre as empresas nessas situações onde ocorre a unilateralidade nas negociações, tendo por resultado um efeito contrário ao desejado, prejudicando a relação de confiança entre as partes do relacionamento.

Faria, Soares e Rocha (2013) consideram a estrutura organizacional do setor industrial automobilístico suficientemente sólida, e nesse contexto a confiança e a abertura de informações é favorecida. No entanto, classificam a prática da $\mathrm{GCl}$, no setor, como tirana, por se tratar de relação onde o fornecedor participa na criação de produtos e contribui nas discussões de custos; no entanto, é a montadora que toma as decisões finais.

Kunimura, Mattos e Scur (2016) perceberam que, apesar das características do OBA torná-lo aparentemente unilateral, o método, se corretamente aplicado, concede à cadeia de suprimento maior eficiência, redução de custos e aumento do nível de confiança mútua.

Outra consideração sobre o OBA é que, além dos aspectos financeiros e de otimização de custos, o processo de abertura de contas também pode gerar benefícios 
Aplicação do Open-Book Accounting (OBA) na perspectiva dos fornecedores de autopeças do

Brasil

Vinícius Almeida Cordeiro, Lara Cristina Francisco de Almeida Fehr, Ana Clara Gama Oliveira, Anderson Martins Cardoso, Marcelo Tavares

socioeconômicos, fato provado pelo Movimento Passe Livre, ocorrido, em 2013, na cidade de São Paulo, que reivindicava a redução da tarifa do transporte público. De acordo com Fernandes, Fernandes e Fernandes (2015), as informações contábeis publicadas nos sites das empresas de transporte foram fundamentais para que se comprovassem os custos efetivos do serviço e a possibilidade de redução do preço da tarifa do transporte urbano público para o consumidor final, já que ficou comprovado não haver uma justificativa plausível para o aumento.

Não é difícil perceber que ainda existe uma série de diferentes definições acerca do OBA. Podemos encontrar pesquisas que consideram o OBA uma ferramenta importante para a construção da confiança entre as partes (Nyaga, Whipple, \& Lynch, 2010) e outras que consideram como pré-requisito a confiança entre as partes para a implementação do sistema (Carr \& Ng, 1995; Kulmala, 2002). De modo geral, verificase que a confiança bilateral é um facilitador do compartilhamento de informações, pois reduz o risco de uso indevido ou oportunista da informação compartilhada (Kulmala \& Kajüter; Valkokari, 2007).

\subsection{Estudos Anteriores}

A quantidade de pesquisas sobre $\mathrm{OBA}$ e $\mathrm{GCl}$ ainda é pequena, mas é possível observar um crescimento de estudos nessa área, visto que, no período de 2011 a 2015, o número de publicações acerca desse assunto foi duas vezes maior que a observada entre os anos de 1992 a 2009 (Santos et al., 2015). Apesar desse crescimento, ainda existem divergências e lacunas nessas pesquisas, mostrando a necessidade de maiores investigações sobre o tema.

Sadeghi e Jokar (2014) realizaram pesquisa cujo objetivo foi identificar e classificar as dimensões do OBA, e conseguiram identificar quatro: (i) natureza e prática de divulgação dos dados contábeis, em que a forma de divulgação é a subdimensão mais importante; (ii) usos dos dados divulgados, em que o aspecto mais relevante é o planejamento e a coordenação dos membros envolvidos; (iii) condições de 
Aplicação do Open-Book Accounting (OBA) na perspectiva dos fornecedores de autopeças do

Brasil

Vinícius Almeida Cordeiro, Lara Cristina Francisco de Almeida Fehr, Ana Clara Gama Oliveira, Anderson

Martins Cardoso, Marcelo Tavares

coordenação do OBA, sendo a confiança o fator mais importante; e (iv) custos de implementação do OBA, em que a subdimensão mais importante é o custo da incerteza relativa ao que ocorrerá quando da aplicação do OBA.

Já Fehr (2015) teve como objetivo investigar, sob a ótica da Economia dos Custos de Transação (ECT), como o OBA e a confiança influenciam a satisfação com o relacionamento entre fornecedores e comprador na cadeia de suprimentos do setor automotivo. Um dos objetivos específicos foi verificar como o OBA vem sendo praticado, sendo correlato ao objetivo da presente pesquisa. Como resultado, encontrou-se que a aplicação desta ferramenta é parcial e forçada, uma vez que é exigência da montadora; ocorre com baixa frequência e de forma unilateral, em que apenas os fornecedores compartilham suas informações.

Os achados ainda mostraram que as principais finalidades de uso do OBA são negociação de preços, identificação de oportunidades de redução de custos e de preços dos produtos. Verificou-se que as informações compartilhadas não se limitam às de custos e ocorrem em diferentes níveis de detalhamento, dependendo do relacionamento. No presente trabalho, pretende-se ampliar a pesquisa de Fehr (2015), investigando agora os fornecedores de autopeças das montadoras, na forma de survey, para ampliar os resultados encontrados.

Abinajm, Faria, Martins e Serio (2015) investigaram a percepção dos gestores das empresas fabricantes de autopeças brasileiras sobre o compartilhamento de informações, a confiança e o comprometimento no relacionamento com algumas montadoras. Os achados mostraram que o compartilhamento é fraco e que os fornecedores identificaram formas diferenciadas de uso da tecnologia de informação (TI), resultando em diferenças significativas nos sistemas utilizados.

Já Di e Wang (2017) pretenderam investigar, via survey, o impacto do OBA no desempenho organizacional. A pesquisa estabelece um modelo que relaciona o OBA e a $\mathrm{GCl}$ com o desempenho: positivo ou negativo. Os resultados mostram que o OBA tem um impacto positivo na gestão da ferramenta $\mathrm{GCl}$, promovendo a melhoria do 
Aplicação do Open-Book Accounting (OBA) na perspectiva dos fornecedores de autopeças do

Brasil

Vinícius Almeida Cordeiro, Lara Cristina Francisco de Almeida Fehr, Ana Clara Gama Oliveira, Anderson Martins Cardoso, Marcelo Tavares

desempenho organizacional. Nesse sentido, concluiu-se que o uso combinado da $\mathrm{GCl}$, do OBA e TI, no gerenciamento dos custos interorganizacionais, melhoram o desempenho da empresa.

A presente pesquisa pretende gerar contribuições complementares, dado que irá verificar como o OBA é aplicado no setor automotivo, como fizeram Abnajm et al. (2015), usando o modelo de survey, na forma de Di e Wang (2017).

\section{METODOLOGIA}

De acordo com o problema de pesquisa, a abordagem é quantitativa, a qual lida com números e usa modelos estatísticos para explicar os resultados. Quanto aos objetivos, é descritiva, pois relata o modo como o OBA vem sendo praticado nas empresas investigadas. Conforme Gil (2010), a pesquisa descritiva mostra as características de determinada população e identifica possíveis relações entre as variáveis. Com relação aos procedimentos técnicos, foi aplicado questionário com perguntas fechadas, via método levantamento ou survey.

O instrumento de coleta de dados teve como base o utilizado por Fehr (2015), estruturado em doze dimensões, ajustado para atender a presente pesquisa. O Quadro 1 , a seguir, exibe as respectivas dimensões para operacionalização do OBA. 
Aplicação do Open-Book Accounting (OBA) na perspectiva dos fornecedores de autopeças do

Vinícius Almeida Cordeiro, Lara Cristina Francisco de Almeida Fehr, Ana Clara Gama Oliveira, Anderson Martins Cardoso, Marcelo Tavares

\begin{tabular}{|c|c|}
\hline $\begin{array}{c}\text { Variáveis a serem } \\
\text { investigadas }\end{array}$ & Detalhamento \\
\hline & 1- Natureza das informações; \\
2- Tipo da informação; \\
& 3 - Frequência do compartilhamento das informações; \\
4 - Nível de detalhe das informações; \\
5 - Direção do compartilhamento das informações; \\
$\begin{array}{c}\text { Dimensões do OBA } \\
\text { (Fehr, 2015) }\end{array}$ \\
6 - Propósitos de uso das informações - perspectiva do comprador; \\
7 - Propósitos de uso das informações - perspectiva do fornecedor; \\
8 - Processos que contempla; \\
9 - Forma de comunicação; \\
10 - Determinantes de custos que contempla; \\
11 - Condições do compartilhamento de informações; \\
12 - Incentivos para o compartilhamento de informações.
\end{tabular}

Quadro 1. Variáveis a serem investigadas

Fonte: Adaptado de Fehr (2015).

O objeto de estudo foi composto pelas empresas da cadeia de suprimentos do setor automotivo do Brasil, especificamente os fornecedores (autopeças) das montadoras de veículos. Os questionários foram criados em formulários da web via aplicação do Google Surveys ${ }^{\circledR}$.

Como critério de inclusão na pesquisa, fizeram parte executivos, diretores e/ou gestores ligados aos Departamentos de Vendas, de Controladoria, de Suprimentos ou de Logística, por se acreditar que possuíam conhecimento adequado sobre a cadeia de suprimentos e o compartilhamento de informações entre as empresas, de forma a garantir confiabilidade das respostas. Não houve identificação das empresas nem dos respondentes.

Foi realizado o levantamento das autopeças e seus respectivos contatos por meio do site da Associação Nacional dos Fabricantes de Veículos Automotores ANFAVEA (2017). Verificou-se que, das autopeças, parte considerável atua apenas no mercado de reposição de peças e, assim, não puderam fazer parte da pesquisa por não se enquadrarem no escopo. Das empresas aptas, foram identificadas, por meio de sindicatos patronais, 245 empresas.

Foi realizado contato telefônico com cada uma das empresas para coleta do email do respondente. Inicialmente, a quantidade de respostas foi baixa. Então foi 
Aplicação do Open-Book Accounting (OBA) na perspectiva dos fornecedores de autopeças do

Brasil

Vinícius Almeida Cordeiro, Lara Cristina Francisco de Almeida Fehr, Ana Clara Gama Oliveira, Anderson Martins Cardoso, Marcelo Tavares

solicitado apoio ao Sindipeças ${ }^{\circledR}$ para enviar os questionários a todas as empresas associadas. Ao fim, foram obtidas 21 respostas, porém somente 17 se relacionavam com montadora de veículos. No total, chegou-se a 16 respostas válidas, considerando as que compartilhavam informações de custos. Os questionários foram enviados entre julho e setembro/2017 e reenviados em fevereiro/2018.

Para análise dos dados, utilizou-se o software Microsoft Excel 2016®.

\section{APRESENTAÇÃO E ANÁLISE dOS RESULTADOS}

\subsection{Análise do Contexto de aplicação do OBA}

De modo geral, a maioria dos respondentes é ligada ao departamento de vendas e/ou comercial. Fehr (2015) constatou que, na perspectiva dos fornecedores do setor automotivo, os contatos com as montadoras, inclusive quanto ao compartilhamento de informações, são realizados por pessoas ligadas a esses departamentos. Quanto ao tempo de empresa informado pelos respondentes, este varia entre um ano e meio a trinta e sete anos. E a maioria deles informou manter relacionamento com montadoras de veículos há mais de dez anos.

Os contratos com as montadoras de todas as empresas analisadas são de fornecimento de peças e componentes. Quanto ao prazo de duração dos contratos, $19 \%$ dos respondentes afirmaram ser mais de 10 anos, 44\% responderam durar entre 5 e 10 anos e $37 \%$ informaram que os contratos variam, em média, entre 1 e 5 anos. Dos fornecedores analisados, $75 \%$ informaram que não iniciaram o compartilhamento de informações desde o início do relacionamento, ou seja, a maior parte teve primeiramente um tempo de relacionamento para, posteriormente, iniciarem esse processo de troca de informações mais estreito. 
Aplicação do Open-Book Accounting (OBA) na perspectiva dos fornecedores de autopeças do Brasil Vinícius Almeida Cordeiro, Lara Cristina Francisco de Almeida Fehr, Ana Clara Gama Oliveira, Anderson Martins Cardoso, Marcelo Tavares

\subsection{Forma de aplicação do OBA conforme suas dimensões}

Nesta seção, serão exibidos os resultados da pesquisa, conforme as 12 dimensões do OBA propostas por Fehr (2015) e detalhados no Quadro 1.

\subsubsection{Quanto à natureza das informações}

Os resultados quanto a essa dimensão foram inconclusivos, pois não foi possível identificar se as informações compartilhadas eram monetárias, físico-operacionais ou ambas.

\subsubsection{Quanto ao tipo de informação compartilhada}

Para esta dimensão, 37,5\% dos respondentes afirmaram que as informações compartilhadas se limitam a custos, e os demais (62,5\%) declararam compartilhar outros tipos de informações, além daquelas relativas a custos. A Figura 1 exibe os resultados quanto aos outros tipos de informações compartilhadas, que não se referem a custos.

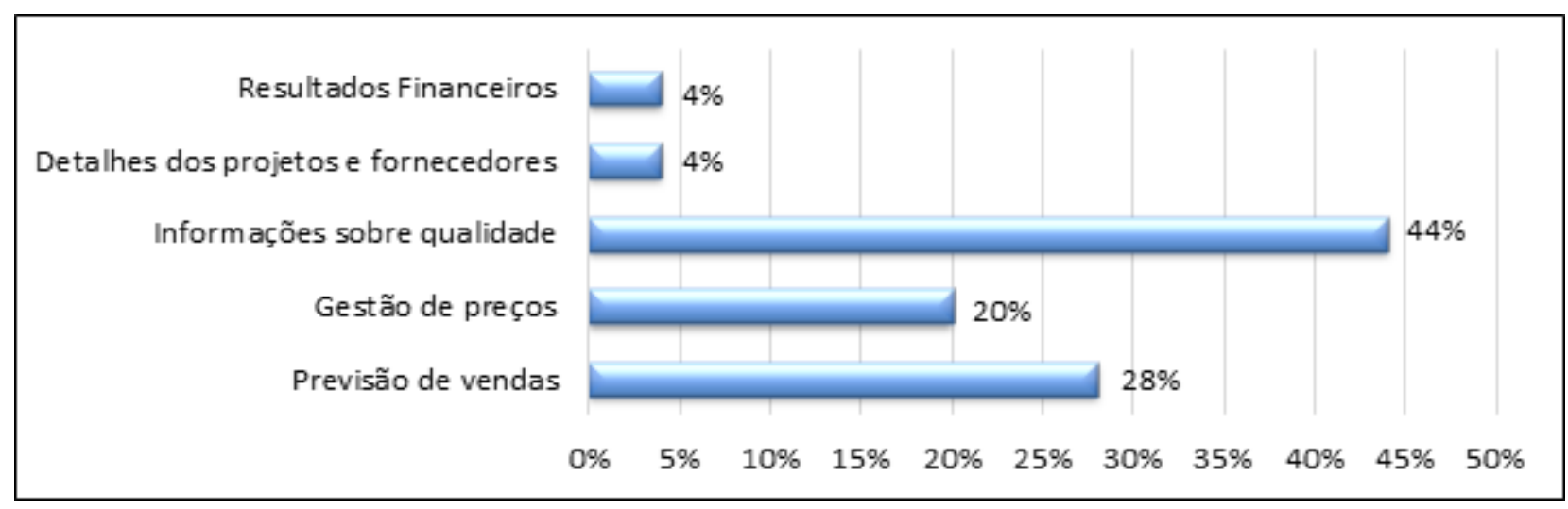

Figura 1. Tipos de informações compartilhadas além das informações de custos.

Fonte: Dados da pesquisa.

Resultados significativos com o modelo R: Previsão de vendas $\times$ Detalhes dos projetos e fornecedores $=$ 0,0206; Previsão de vendas $x$ Resultados Financeiros = 0,0206; Informações sobre qualidade $x$ Detalhes dos projetos e fornecedores $=0,0009$; Informações sobre qualidade $\times$ Resultados financeiros $=0,0009$. 
Aplicação do Open-Book Accounting (OBA) na perspectiva dos fornecedores de autopeças do

Verifica-se, na Figura 1, que, para os fornecedores que divulgam outros tipos de informações, além das referentes aos custos, as mais compartilhadas referem-se à qualidade e à previsão de vendas, seguidas da gestão de preços.

\subsubsection{Quanto à frequência do compartilhamento das informações}

A frequência do compartilhamento de informações é exibida na Figura 2.

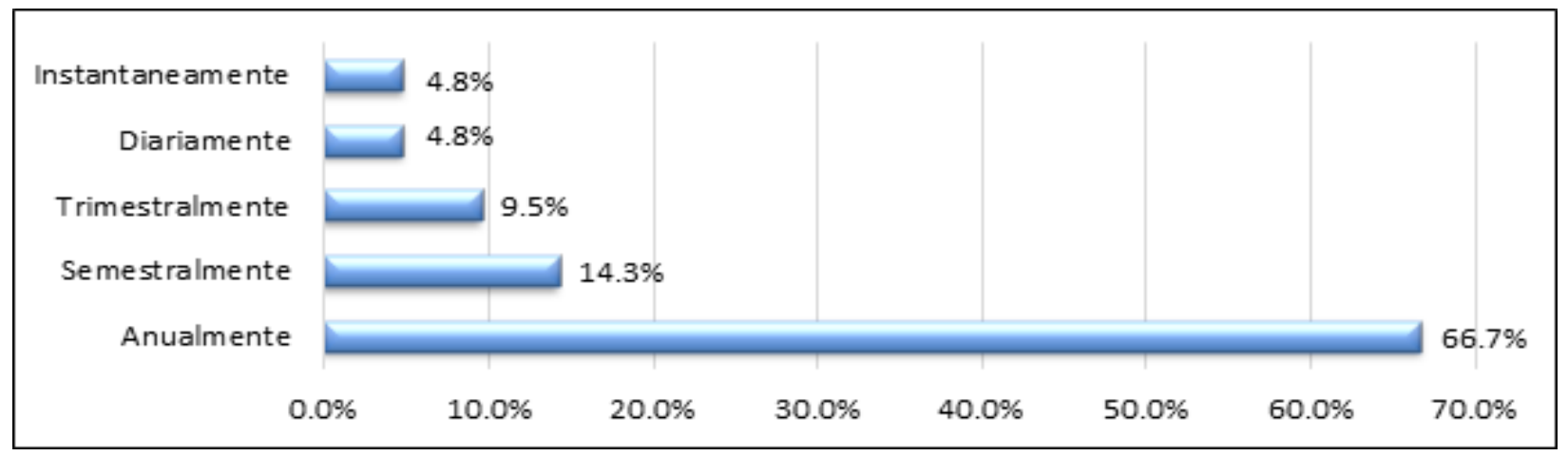

Figura 2. Frequência de compartilhamento.

Fonte: Dados da pesquisa.

Resultados significativos com o modelo R: Anualmente x Semestralmente $=0,0005$; Anualmente $x$ Trimestralmente $=0,0001$; Anualmente $\mathrm{x}$ Diariamente $=0,0000 ;$ Anualmente $\mathrm{x}$ Instantaneamente $=$ 0,0000 .

Verifica-se, pela Figura 2, que a frequência do compartilhamento de informações é baixa e ocorre, em quase $70 \%$ dos casos, anualmente. Poucas empresas compartilham suas informações com maior frequência. $A$ anualidade do compartilhamento de informações ocorre pela necessidade de ajuste de preços, prevista contratualmente.

\subsubsection{Quanto ao nível de detalhe das informações}

Outra dimensão do OBA refere-se ao nível de detalhe das informações compartilhadas. Conforme Fehr (2015), o nível de detalhamento de informações está relacionado ao tipo e à natureza da informação e à sua agregação ou não, o que pode indicar níveis baixos, médios e altos de detalhe das informações.

Para esta dimensão, a Figura 3 mostra o nível de detalhe dessas informações 
Aplicação do Open-Book Accounting (OBA) na perspectiva dos fornecedores de autopeças do

Brasil

Vinícius Almeida Cordeiro, Lara Cristina Francisco de Almeida Fehr, Ana Clara Gama Oliveira, Anderson Martins Cardoso, Marcelo Tavares

nas empresas objeto de estudo.

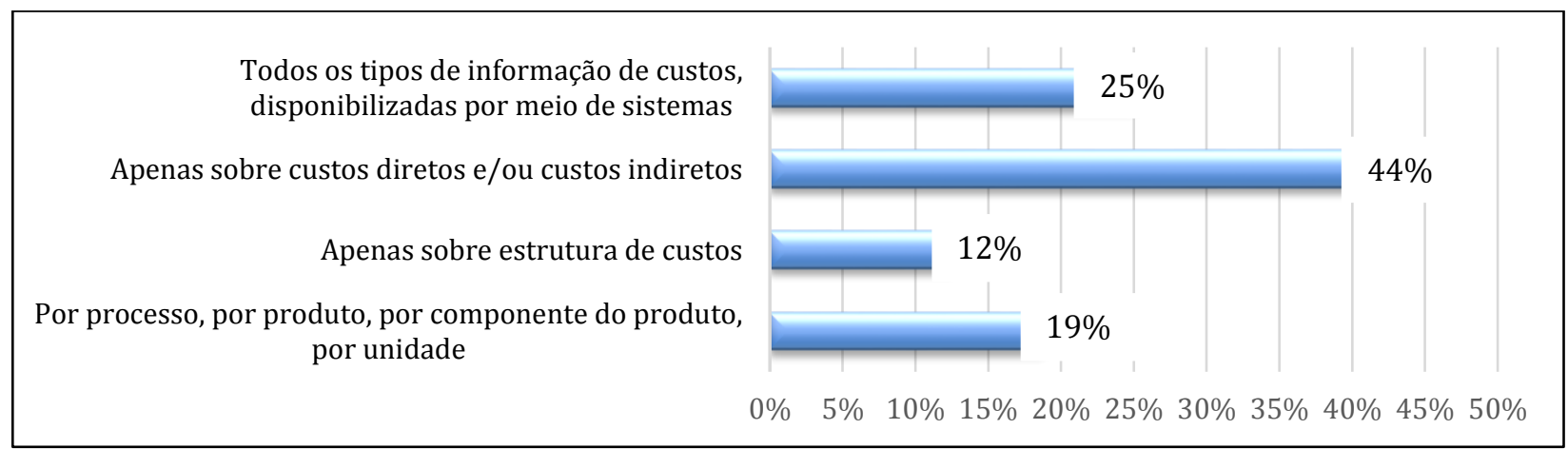

Figura 3. Nível de detalhe das informações compartilhadas.

Fonte: Dados da pesquisa.

Não houve resultado significativo de acordo com o modelo $R$.

Cerca de $44 \%$ dos respondentes afirmaram compartilhar apenas informações sobre custos diretos e indiretos, e em torno de 12\% afirmaram compartilhar somente informações sobre estrutura de custos, somando um total de $56 \%$, ou seja, a maior parte das empresas apresenta baixo detalhamento de informações compartilhadas.

Ao mesmo tempo, parte dos respondentes declarou divulgar as informações de maneira mais detalhada (Figura 3): 25\% afirmaram compartilhar todos os tipos de informações de custos por meio de sistemas integrados e 19\% compartilham informações por processo, por produto, por componente do produto e/ou por unidade.

\subsubsection{Quanto à direção do compartilhamento das informações}

Esta dimensão mostra em qual direção o compartilhamento de informações ocorre, que, segundo Fehr (2015), pode ser: unilateral - no sentido do fornecedor para o comprador ou no sentido do comprador para o fornecedor; bilateral - tanto o fornecedor quanto o comprador compartilham suas informações; ou multilateral - tanto o comprador quanto os fornecedores tier 1, tier 2 etc. divulgam suas informações de custos. A Figura 4 exibe os resultados para as empresas analisadas. 
Aplicação do Open-Book Accounting (OBA) na perspectiva dos fornecedores de autopeças do

Vinícius Almeida Cordeiro, Lara Cristina Francisco de Almeida Fehr, Ana Clara Gama Oliveira, Anderson Martins Cardoso, Marcelo Tavares

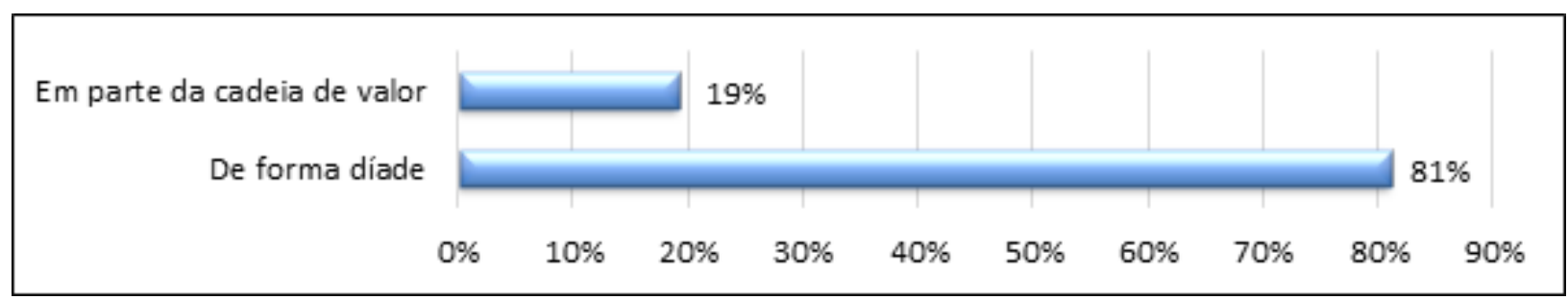

Figura 4. Direção do compartilhamento das informações.

Fonte: Dados da pesquisa.

Resultado significativo de acordo com o modelo $\mathrm{R}$ : De forma díade $\times$ Em parte da cadeia de valor $=$ 0,0001 .

Percebe-se, pela Figura 4, que a grande maioria das empresas pesquisadas $(81 \%)$ compartilham suas informações de maneira unilateral, no sentido do fornecedor para o comprador, confirmando a literatura sobre OBA no setor automotivo.

\subsubsection{Propósitos de uso das informações - na perspectiva da montadora e dos fornecedores \\ Essas dimensões não se aplicam a esta pesquisa, visto que não foi realizada} com as montadoras de veículos, apenas com os fornecedores. Também as montadoras não compartilham tais informações com os fornecedores.

\subsubsection{Quanto aos processos que contempla}

Esta dimensão tem o intuito de verificar quais processos as informações compartilhadas contemplam, a saber: projeto do produto, pré-produção, produção em curso, comercialização, logística e processos administrativos. A Figura 5 exibe os processos contemplados nas informações compartilhadas pelas autopeças investigadas. 
Aplicação do Open-Book Accounting (OBA) na perspectiva dos fornecedores de autopeças do

Vinícius Almeida Cordeiro, Lara Cristina Francisco de Almeida Fehr, Ana Clara Gama Oliveira, Anderson Martins Cardoso, Marcelo Tavares

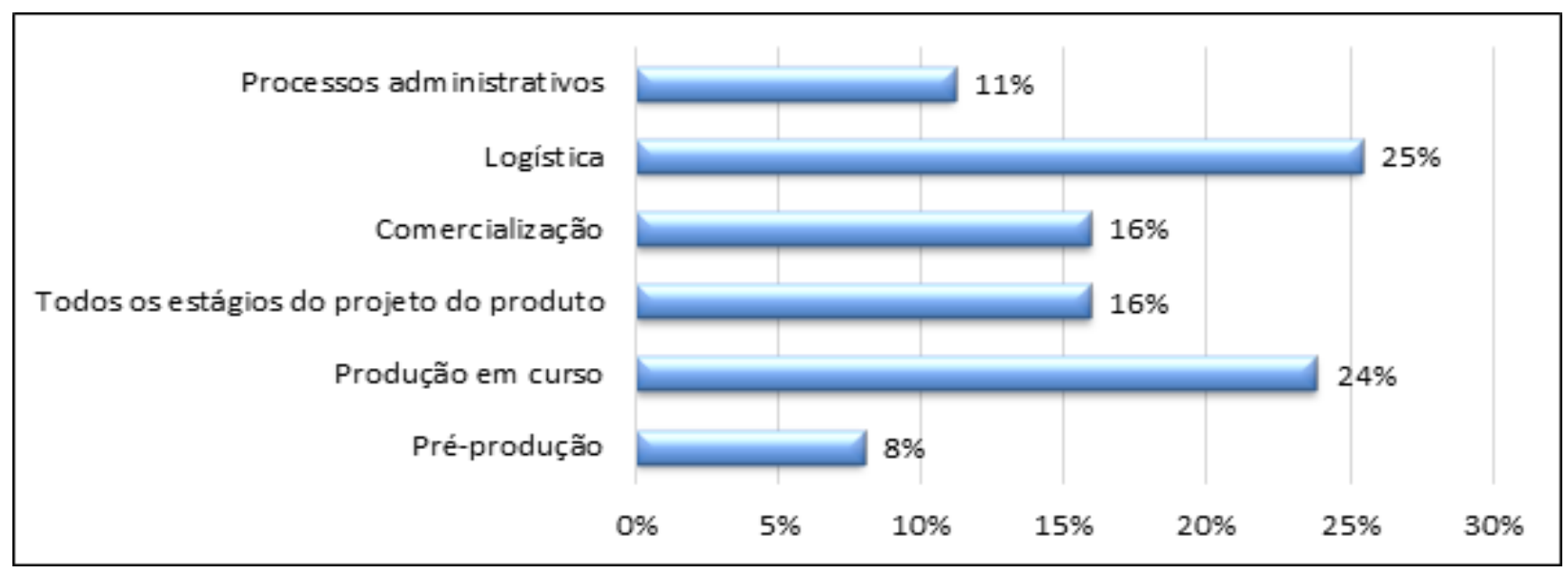

Figura 5. Processos contemplados nas informações compartilhadas.

Fonte: Dados da Pesquisa.

Resultados significativos com o modelo R: Logística x Pré-produção $=0,0221$; Pré-produção x Produção em curso = 0,0221; Produção em curso x Processos administrativos $=0,0196$.

A Figura 5 mostra que a maior parte dos processos contemplados nas informações compartilhadas refere-se à logística, à produção em curso e à comercialização, ainda que as empresas divulguem informações não apenas sobre um processo, mas a outros também, ao mesmo tempo. Em tese, os processos que deveriam ser mais contemplados nessas informações são projetos do produto e préprodução, pois são nesses, principalmente no projeto do produto, que se pode aplicar uma gestão mais detalhada dos custos interorganizacionais e com maiores potenciais de redução e/ou otimização de custos.

\subsubsection{Quanto à forma de comunicação}

A forma de comunicação é outra dimensão considerada no OBA e evidencia de que forma ocorre a comunicação das informações compartilhadas nas empresas estudadas. Conforme a Figura 6, o compartilhamento ocorre mais via sistemas, sendo que muitos fornecedores afirmaram ocorrer por mais de uma forma, por exemplo: impressa e via e-mail ou oral, impressa e via sistemas. 
Aplicação do Open-Book Accounting (OBA) na perspectiva dos fornecedores de autopeças do

Vinícius Almeida Cordeiro, Lara Cristina Francisco de Almeida Fehr, Ana Clara Gama Oliveira, Anderson Martins Cardoso, Marcelo Tavares

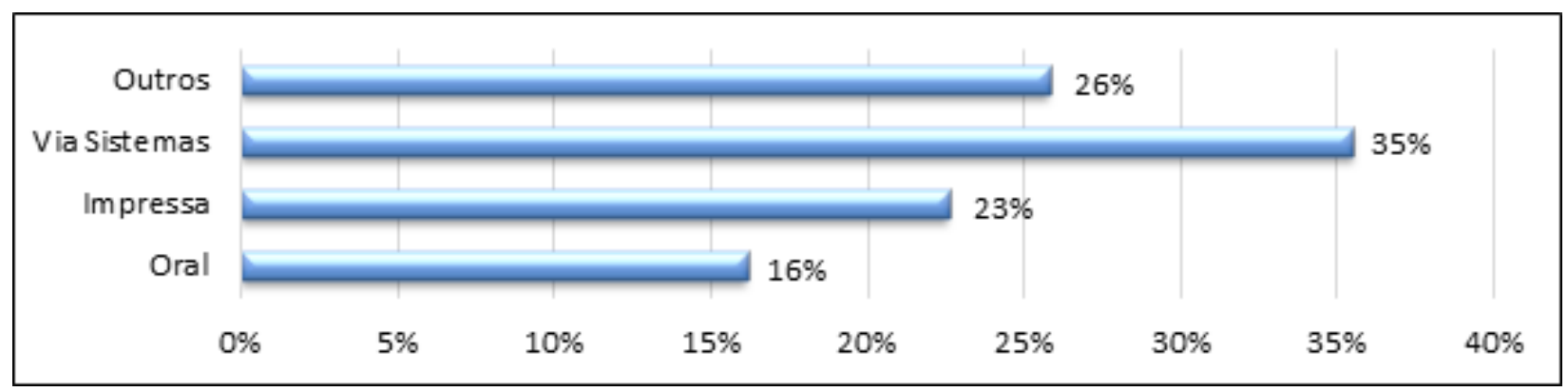

Figura 6. Forma de comunicação das informações compartilhadas.

Fonte: Dados da pesquisa.

* Não houve comparabilidade significativa entre os quatro sistemas conforme o modelo R.

A forma "outros" engloba planilhas, formulários específicos e via e-mail, acrescentadas pelos próprios respondentes. Nesse quesito, a indústria ainda se encontra atrasada quanto ao uso de sistemas de informação, comparativamente ao varejo/serviços.

\subsubsection{Quanto aos determinantes de custos que contemplam}

Esta é uma dimensão que serve para verificar quais determinantes de custos são contemplados nas informações compartilhadas, o que daria condições para a gestão de custos entre as empresas. De acordo com Costa (2011), são os determinantes que mais influenciam as estruturas de custos das empresas. Assim, se as empresas pretendem reduzir e/ou otimizar seus custos, deveriam compartilhar essas informações de forma contínua.

A Figura 7 exibe os determinantes contemplados nas informações compartilhadas pelas empresas em análise. 
Aplicação do Open-Book Accounting (OBA) na perspectiva dos fornecedores de autopeças do

Brasil

Vinícius Almeida Cordeiro, Lara Cristina Francisco de Almeida Fehr, Ana Clara Gama Oliveira, Anderson Martins Cardoso, Marcelo Tavares

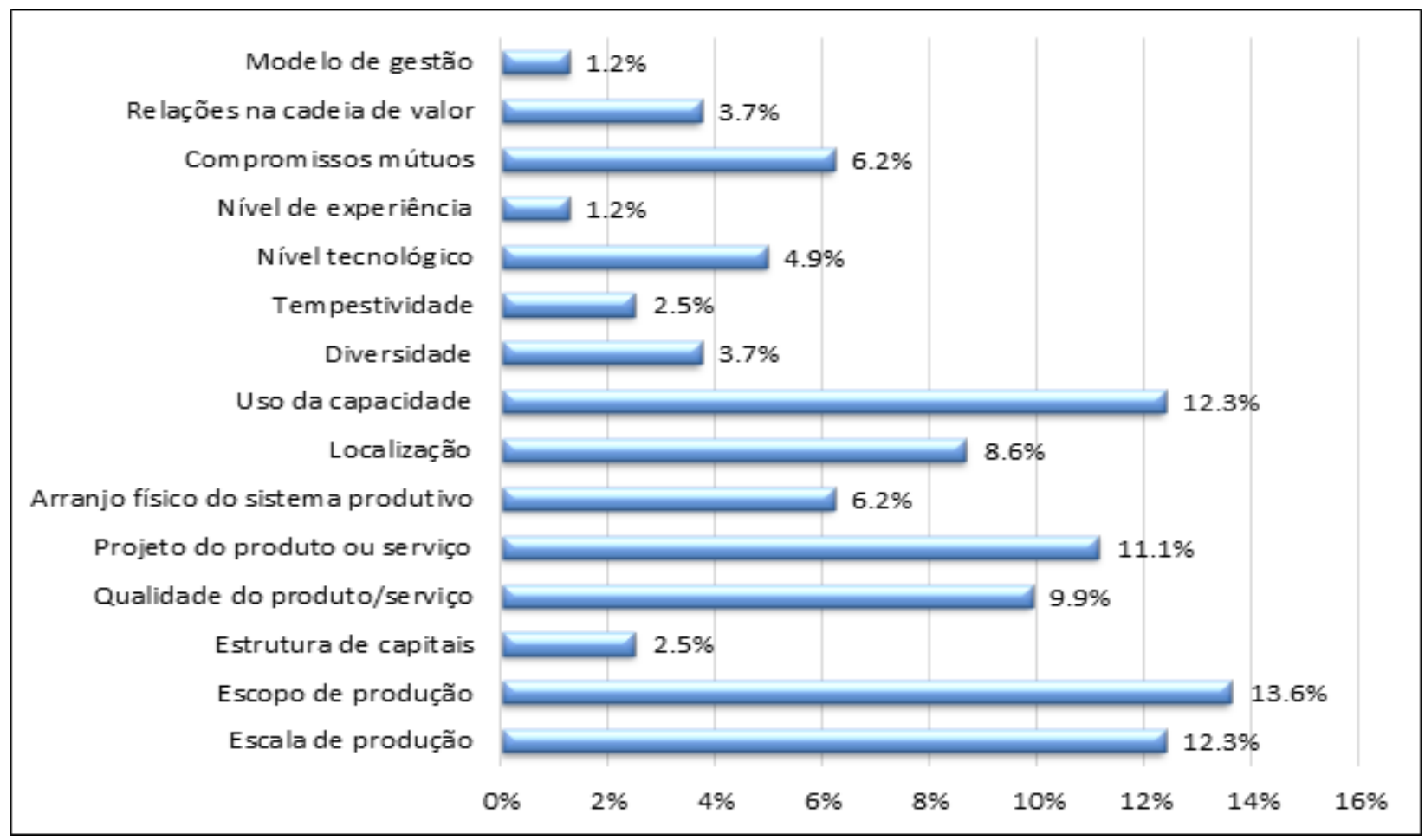

Figura 7. Determinantes de custos contemplados nas informações compartilhadas.

Fonte: Dados da pesquisa.

Resultados significativos com o modelo R: Escala de produção $x$ Estrutura de capitais $=0,0164$; Escala de produção $\times$ Diversidade $=0,0429$; Escala de produção $\times$ Tempestividade $=0,0164$; Escala de produção x Nível de experiência = 0,0049; Escala de produção x Relações na cadeia de valor =0,0429; Escala de produção $\times$ Modelo de gestão = 0,0049; Escopo de produção x Estrutura de capitais = 0,0092; Escopo de produção $\times$ Diversidade $=0,0253$; Escopo de produção $\times$ Tempestividade $=0,0092$; Escopo de produção $\times$ Modelo de gestão $=0,0027$; Estrutura de capital $\times$ Projeto do produto ou serviço $=0,0288$; Estrutura de capital $x$ Uso da capacidade $=0,0164$; Qualidade do produto/serviço x Nível de experiência = 0,0164; Escopo de produção x Nível de experiência = 0,0027; Escopo de produção x Relações na cadeia de valor = 0,0253; Qualidade do produto/serviço $\times$ Modelo de gestão $=0,0164$; Projeto do produto ou serviço $\times$ Tempestividade $=0,0288$; Projeto do produto ou serviço $\times$ Nível de experiência $=0,0090$; Projeto do produto ou serviço $\times$ Relações na cadeia de valor = 0,0090; Localização x Nível de experiência = 0,0296; Localização $\times$ Modelo de gestão = 0,0296; Uso da capacidade $\times$ Diversidade $=0,0429$; Uso da capacidade $\mathrm{x}$ Tempestividade $=0,0164$; Uso da capacidade $\mathrm{x}$ Nível de experiência $=0,0049$; Uso da capacidade $\times$ Relações na cadeia de valor $=0,0429$; Uso da capacidade $\times$ Modelo de gestão $=0,004$.

Verifica-se, na Figura 7, que o determinante de custos mais considerado nas informações compartilhadas é o escopo da produção seguido de escala de produção. Ressalta-se que a maioria dos respondentes afirmou ter contemplado, em conjunto, vários determinantes de custos nas informações compartilhadas. Apenas 25\% 
Aplicação do Open-Book Accounting (OBA) na perspectiva dos fornecedores de autopeças do Brasil Vinícius Almeida Cordeiro, Lara Cristina Francisco de Almeida Fehr, Ana Clara Gama Oliveira, Anderson Martins Cardoso, Marcelo Tavares

declararam contemplar somente um determinante de custos, sobressaindo, nesses casos, o escopo de produção.

\subsubsection{Quanto às condições em que ocorre o compartilhamento de informações}

Para essa dimensão, a Figura 8, a seguir, mostra em que condições o OBA é aplicado. Nota-se que a condição principal para o compartilhamento das informações de custos é ser uma exigência das montadoras. Muitos respondentes marcaram mais de uma opção para as condições do compartilhamento, por exemplo, indicaram ser uma exigência das montadoras e que, apesar de não haver confiança ou de não haver cooperação, era importante para os negócios.

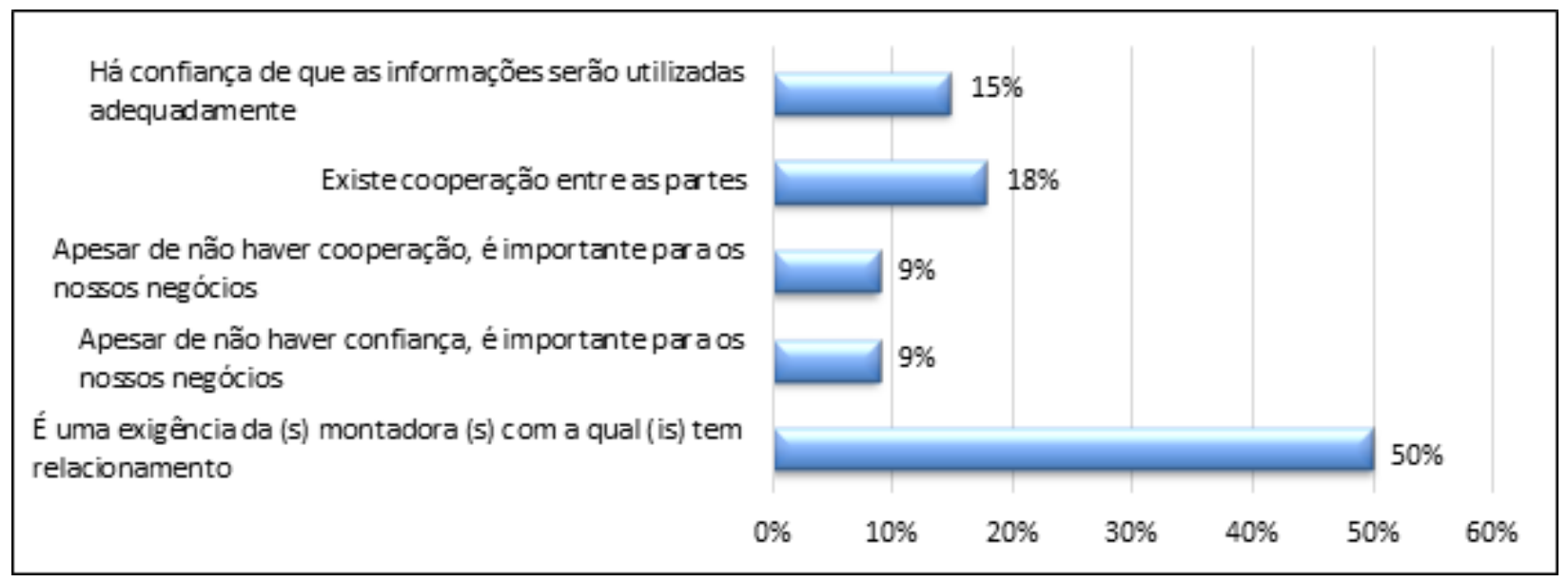

Figura 8. Condições em que ocorre o compartilhamento de informações.

Fonte: Dados da pesquisa.

O resultado evidenciado de que o compartilhamento ocorre por ser uma exigência das montadoras corrobora a literatura de OBA na indústria automotiva (Fehr, 2015; Abinajm-Filho, 2011). Uma minoria indicou existir cooperação e confiança de que as informações serão utilizadas adequadamente, conforme relataram Shen, Wang e Teng (2017). 
Aplicação do Open-Book Accounting (OBA) na perspectiva dos fornecedores de autopeças do

Brasil

Vinícius Almeida Cordeiro, Lara Cristina Francisco de Almeida Fehr, Ana Clara Gama Oliveira, Anderson

Martins Cardoso, Marcelo Tavares

\subsubsection{Quanto aos incentivos para o compartilhamento}

Para esta dimensão, todos os respondentes declararam não haver incentivos por parte das montadoras, para o compartilhamento de informações de custos.

\section{SÍNTESE DOS RESULTADOS}

Verificou-se, na perspectiva dos fornecedores, que o OBA é aplicado unilateralmente, no sentido do fornecedor para a montadora e com baixa frequência. Este aspecto pode sugerir que as informações não são usadas para gestão de custos interorganizacionais e, sim, negociação de preço e identificação de oportunidades de redução de custos dos produtos fornecidos, o que corrobora os achados de Fehr (2015).

As informações compartilhadas envolvem outros tipos de informações, além das de custos, e o nível de detalhamento, na maior parte dos casos, é baixo ou pouco detalhado. De acordo com Kajüter e Kulmala (2005), por vezes, fornecedores podem não ter condições de compartilhar suas informações ou as compartilham a um nível baixo de detalhe, por não conseguirem gerar informações de custos a um nível satisfatório aos compradores.

O OBA é aplicado de maneira forçada, já que os fornecedores declararam compartilhar informações por ser uma exigência das montadoras, prática comum do setor. O OBA não é aplicado espontaneamente pelas autopeças, nem é pautado na confiança. Sobre o tema, Sadeghi e Jokar (2014) mostram que a confiança é um dos aspectos mais importantes para a aplicação do OBA e, para Shen, Wang e Teng (2017), muitos relacionamentos não conseguem atingir os resultados esperados devido aos problemas de coordenação e desalinhamento de ações ocasionados pelas limitações cognitivas dos parceiros em não saberem como os membros da rede irão se comportar em situações de interdependência. 
Aplicação do Open-Book Accounting (OBA) na perspectiva dos fornecedores de autopeças do

Brasil

Vinícius Almeida Cordeiro, Lara Cristina Francisco de Almeida Fehr, Ana Clara Gama Oliveira, Anderson

Martins Cardoso, Marcelo Tavares

Mesmo havendo vários tipos de incentivos, tais como suporte na negociação de preços com outras empresas, estabelecimento de benefícios mútuos ("ganha-ganha”), garantia de contrato futuro, otimização do tempo nas negociações, aumento do volume de negócios mútuos das firmas e contratos mais longos, resolução de problemas em conjunto, etc., todos os respondentes indicaram não haver incentivos por parte das montadoras para o compartilhamento de informações de custos. Esse resultado contradiz a literatura acerca do OBA, pois geralmente existe algum tipo de incentivo oferecido.

Outro aspecto verificado refere-se ao fato de que a maioria dos respondentes alegou que o processo de compartilhamento de informações de custos é praticado de diferentes formas, dependendo da montadora com a qual estão se relacionando. Esse achado pode estar relacionado a fatores como o tipo de produto fornecido, ao porte dessas empresas, ao nível de dependência entre elas, à confiança estabelecida, aos propósitos de uso das informações que inclui também o oportunismo por parte da montadora, dentre outros.

Por fim, verifica-se que a prática do OBA, na perspectiva dos fornecedores de autopeças, mostra-se fraca, conforme identificou Abnajm et al. (2015). Nessas condições, a forma como é aplicado - unilateralmente - pode comprometer os potenciais benefícios da ferramenta, justamente pelo risco de comportamento oportunista no uso das informações. Isso impacta, inclusive, no nível de detalhamento e na frequência de compartilhamento, dificultando uma adequada gestão de custos entre as empresas.

\section{CONSIDERAÇÕES FINAIS}

Esta pesquisa teve como objetivo identificar a extensão na qual o OBA vem sendo aplicado na perspectiva dos fornecedores de autopeças do Brasil. Os resultados evidenciaram que o OBA é aplicado de maneira unilateral, com baixo nível de detalhamento das informações e baixa frequência na maior parte dos casos. 
Aplicação do Open-Book Accounting (OBA) na perspectiva dos fornecedores de autopeças do Brasil Vinícius Almeida Cordeiro, Lara Cristina Francisco de Almeida Fehr, Ana Clara Gama Oliveira, Anderson Martins Cardoso, Marcelo Tavares

A maioria dos processos contemplados nas informações refere-se à logística, à produção em curso e à comercialização. Todavia, esperava-se que os dados mais compartilhados fossem quanto ao projeto do produto e pré-produção, para que se pudesse aplicar uma adequada gestão de custos entre as empresas, para se ter maiores potenciais de geração de benefícios.

Verificou-se, ainda, que a forma de comunicação dominante é via sistemas, também impressa e via e-mail. São compartilhados outros tipos de informações, além das de custos. Quanto às condições do compartilhamento, alegaram exigência prevista em contrato, não sendo, portanto, espontânea. A maior parte dos fornecedores informou que não iniciaram o compartilhamento de informações já no início do relacionamento; tiveram primeiro um tempo de relacionamento para depois iniciarem esse processo, salvaguardando-se por meio de contrato.

Os achados evidenciaram também que não há incentivo para o compartilhamento de informações, o que não está alinhado com a literatura na área, visto que, geralmente, existe algum tipo de incentivo para a prática do OBA.

Assim, o OBA mostra-se um processo fraco no setor automotivo. Aplicado de maneira unilateral, gera desconfiança e risco de comportamento oportunista, o que pode afetar as dimensões do OBA propostas. As empresas perdem a chance de aplicar uma adequada gestão de custos interorganizacionais que os ajudaria a gerar e/ou manter vantagem competitiva.

Como limitação do estudo, tem-se a baixa taxa de resposta, não permitindo a generalização. Para futuras pesquisas, sugere-se identificar os fatores do contexto relacional do OBA mais representativos na sua aplicação. Pode-se também realizar esta pesquisa em outros setores da economia e, ainda, pesquisar duas ou mais montadoras, de tamanhos diferentes, e alguns fornecedores diretos em comum, para verificar se surgem resultados diferentes. 
Aplicação do Open-Book Accounting (OBA) na perspectiva dos fornecedores de autopeças do

Brasil

Vinícius Almeida Cordeiro, Lara Cristina Francisco de Almeida Fehr, Ana Clara Gama Oliveira, Anderson Martins Cardoso, Marcelo Tavares

\section{REFERÊNCIAS}

Abinajm Filho, J. (2011). Confiança, Comprometimento e Efeito Chicote na Gestão da Cadeia de Suprimentos Automotiva. (Dissertação de Mestrado em Administração), Faculdade de Administração, Univ. Mun. de São Caetano do Sul, São Caetano do Sul.

Abinajm Filho, J., Faria, A. C. de, Martins, D. M., \& Serio, L. C. D. (2015). Information Sharing, Trust and Commitment: Perception of Brazilian Autoparts on Automakers of 'Various Ethnic Groups. Brazilian Business Review, 12(6), 1-23.

Aguiar, A. B. de, Rezende, A. J., \& Rocha, W. (2008). Uma análise da complementaridade entre gestão interorganizacional de custos e Open-Book Accounting. Base - Revista de Administração e Contabilidade da Unisinos, 5(1), 6676.

Associação Nacional dos Fabricantes de Veículos Automotores [ANFAVEA]. (2017). Anuário da Indústria Automobilística Brasileira. Recuperado de: <http://www.anfavea.com.br/anuario.html>. Acesso em: 18/dez./2017.

- (2018). Anuário da Indústria Automobilística Brasileira. Recuperado de: <http://www.anfavea.com.br/anuario.html>. Acesso em: 21/mar./2018.

Bhimani, A., Souza, B. C., \& Rocha, W. (2011, nov./dec.). Inter-organizational cost management in a Brazilian context. Cost Management, 5-16. Recuperado de: $<$ http://blog-fipecafi.imprensa.ws/wp-content/uploads/2012/04/Blog-Professores-da-

FIPECAFI-publicam-artigo-na-Cost-Management-Magazine.pdf>. Acesso em: 03/dez./2017.

Camacho, R. R. (2010). Fatores condicionantes da gestão de custos interorganizacionais na cadeia de valor de hospitais privados no Brasil: uma abordagem à luz da Teoria da Contingência. (Tese de Doutorado em Controladoria), Faculdade de Economia, Administração e Contabilidade da Universidade de São Paulo, São Paulo.

Costa, R. M., \& Henkin, H. (2016). Estratégias competitivas e desempenho da indústria automobilística no Brasil. Economia e Sociedade, 25(2), 457-487.

Costa, S. A. (2011). Análise de custos de concorrentes: um estudo dos determinantes de custos no setor de eletroeletrônicos. São Paulo, 196 f. Dissertação (Mestrado em Ciências Contábeis) - Faculdade de Economia, Administração e Contabilidade da 
Aplicação do Open-Book Accounting (OBA) na perspectiva dos fornecedores de autopeças do

Brasil

Vinícius Almeida Cordeiro, Lara Cristina Francisco de Almeida Fehr, Ana Clara Gama Oliveira, Anderson Martins Cardoso, Marcelo Tavares

Universidade de São Paulo.

DhaifAllah, B., Md Auzair, S., Maelah, R., \& Ismail, M. (2016). Inter-Organizational Cost Management and Open Book Accounting: A Review. Asian Journal Of Accounting Perspectives, 9(1), 67-96. Recuperado de: $<$ http://ajap.um.edu.my/index.php/AJAP/article/view/7269>. Acesso em: 06/dez./2017.

Di, W., \& Wang, S. (2017). Empirical research on the impact of open-book accounting on organizational performance. Research on Modern Higher Education, 2, 199-204.

Ellström, D., \& Larsson M. H. Dynamic and static pricing in open-book accounting. (2017). Qualitative Research in Accounting \& Management, 14(1), 21-37. Recuperado de: <https://doi.org/10.1108/QRAM-09-2015-0071>. Acesso em: 18/abr./2017.

Faria, A. C., Soares, I. C., Rocha, W., \& Rossi, G. B. (2013). The Adoption of Interorganizational cost Management in a Vehicle Assembly plant in the Greater Region of ABC. Revista Brasileira de Gestão e Negócios, (15)49, 617-638. Recuperado de: <http://www.redalyc.org/articulo.oa?id=94729777008>. Acesso em: 18/abr./2017.

Fehr, L. C. F. A. (2015). Open-Book Accounting e confiança: influência sobre a satisfação com o relacionamento entre comprador e fornecedores no setor automotivo do Brasil. (Tese de Doutorado em Contabilidade). Faculdade de Economia, Administração e Contabilidade, Universidade de São Paulo, São Paulo.

Fernandes, B. A. O. F., Fernandes, J. L. N., \& Fernandes, J. W. N. (2015). O movimento passe livre e a contabilidade de livros abertos (Open-book Accounting). Revista FAE, 18(1), 180-195. Recuperado de: <https://revistafae.fae.edu/revistafae/article/view/39/39 >. Acesso em: 20/maio/2017.

Gil, A. C. (2010). Como elaborar projetos de pesquisa. (5a ed). São Paulo: Atlas.

Grafton, J., \& Mundy, J. (2017). Relational contracting and the myth of trust: Control in a co-opetitive setting. Management Accounting Research, 36, 24-42. Recuperado de: <https://doi.org/10.1016/j.mar.2016.07.008. Acesso em: 18/out./2017.

Kulmala, H. I., Paranko, J., \& Uusi-Rauva, E. (2002). The role of cost management in network relationships. International Journal of Production Economics, 79(1), 33-43. Recuperado de: <https://doi.org/10.1016/S0925-5273(00)00061-X>. Acesso em: 19/jun./2017. 
Aplicação do Open-Book Accounting (OBA) na perspectiva dos fornecedores de autopeças do

Brasil

Vinícius Almeida Cordeiro, Lara Cristina Francisco de Almeida Fehr, Ana Clara Gama Oliveira, Anderson Martins Cardoso, Marcelo Tavares

Kajüter, P., \& Valkokari, K. (2007). Inter-organizational cost management in SME networks. $8^{\text {th }}$ Manufacturing Accounting Research, Cost and Performance Management in Services and Operations, Trento. Recuperado de: $<$ https://www.researchgate.net/profile/Katri_Valkokari/publication/38288569_Interorganizational_cost_management_in_SME_networks/links/Ofcfd50efb81d5aac4000 000/Inter-organizational-cost-manāgement-in-SME-networks.pdf>. Acesso em: 19/jun./2017.

Kunimura, A. A., Mattos, C. A., \& Scur, G. (2016). Open-Book Accounting: estudo de caso em uma empresa farmacêutica. $36^{\text {th }}$ Encontro Nacional de Engenharia de Produção - ENEGEP, João Pessoa, Brasil. Recuperado de: <http://www.abepro.org.br/biblioteca/TN_STO_228_331_29215.pdf>. Acesso em: 19/abr./2018.

Marini, M. L. (2003). O relacionamento e as novas configurações entre montadoras de automóveis e seus fornecedores. (Dissertação de Mestrado em Engenharia da Produção), Universidade Federal de Santa Catarina, Florianópolis.

Martins, R. S., Souza-Filho, O. V., Pereira, S. C. F., \& Di Serio, L. C. (2012). Collaborative practices in the management of the brazilian automotive chain: does the origin of automakers matter? 23 th Production and Operations Management Society - POMS Annual Conference, Chicago, Illinois.

., Souza Filho, O. V., \& Pereira, S. C. F. (2012). Alinhamento estratégico nas cadeias de suprimento da indústria automobilística brasileira. REAd. Revista Eletrônica de Administração, 18(3), 581-606. Recuperado em: <http://dx.doi.org/10.1590/S1413-23112012000300001>. Acesso em: 09/abr./2018.

Mouritsen, J., Hansen, A., \& Hansen C. O. (2001). Inter-organizational controls an organizational competences: episodes around target cost management, functional analysis and open book accounting. Management Accounting Research, (12), 221244.

Sadeghi, L., \& Jokar, I. (2014). Identification and classification of open book accounting dimensions by considering inter-organizational cost management: a case study of petrochemical companies listed in Tehran Stock Exchange. Management Science Letters, 4(2), 295-302. Recuperado de: <http://www.growingscience.com/msl/Vol4/msl_2013_399.pdf>. Acesso em: 19/maio/ 2017.

Santos, A. M. M. M. (2001, sep.). Reestruturação da indústria automobilística na 
Aplicação do Open-Book Accounting (OBA) na perspectiva dos fornecedores de autopeças do

Brasil

Vinícius Almeida Cordeiro, Lara Cristina Francisco de Almeida Fehr, Ana Clara Gama Oliveira, Anderson Martins Cardoso, Marcelo Tavares

América do Sul. BNDES Setorial, 14, 47-63. Recuperado de: <https://web.bndes.gov.br/bib/jspui/handle/1408/4381>. Acesso em: 20/jun./2017.

Santos, E. F. et al. (2015). Gestão de Custos Interorganizacionais e Open-book Accounting: um estudo bibliométrico das publicações nacionais e internacionais. Congresso Brasileiro de Custos - CBC, Foz do Iguaçu, Paraná, 22.

Serviço Brasileiro se Apoio às Micro e Pequenas Empresas (SEBRAE). (2015). Boletim Inteligência. Recuperado de: <http://www.bibliotecas.sebrae.com.br/chronus/ARQUIVOS_CHRONUS/bds/bds.nsf /f9087ff9c7f6da378eb8fb1f151fc79e/\$File/5792.pdf>. Acesso em: 16/set./2016.

Shen, L., Wang, Y., \& Teng, W. (2017). The moderating effect of interdependence on contracts in achieving equity versus efficiency in interfirm relationships. Journal of Business Research, 78, 277-284.

Souza, B. C. (2008). Fatores determinantes da gestão de custos interorganizacionais. (Dissertação de Mestrado em Contabilidade). Faculdade de Economia, Administração e Contabilidade, Universidade de São Paulo, São Paulo.

, \& Rocha, W. (2009). Gestão de custos interorganizacionais: ações coordenadas entre clientes e fornecedores para otimizar resultados. São Paulo: Atlas.

Suomala, P., Lahikainen, T., Lyly-Yrjänäinen, J., \& Paranko, J. (2010) Open book accounting in practice - exploring the faces of openness. Qualitative Research in Accounting \& Management, 7(1),71-96. Recuperado de: <https://doi.org/10.1108/11766091011034280>. Acesso em: 09/abr./2018.

Vanalle, R. M., \& Salles, J. A. A. (2011). Relação entre montadoras e fornecedores: modelos teóricos e estudos de caso na indústria automobilística brasileira. Gestão \& Produção, 18(2), 237-250. Recuperado de: <http://www.scielo.br/pdf/gp/v18n2/02.pdf>. Acesso em: 21/fev./2018.

Data de Submissão: 09/12/2019 Data de Aceite: 17/12/2019 